\title{
Ocean anoxia and the emergence of ferruginous and euxinic states through Earth's history
}

\begin{abstract}
SEAN A. CROWE
${ }^{1}$ Division of Earth and Planetary Sciences, The University of Hong Kong, Hong Kong SAR, sacrowe@hku.hk

The oceans were pervasively anoxic for more than two thirds of Earth's history and widespread marine oxygenation is a relatively recent $(<500 \mathrm{Ma})$ phenomenon. Even in the last $500 \mathrm{Ma}$, however, the oceans experienced episodes of strong deoxygenation, so termed ocean anoxic events (OAEs), during which regions of anoxia expanded in association with enhanced volcanism, climate perturbations, and biological crises. Today, the oceans are experiencing rapid deoxygenation due to the combined effects of climate warming and anthropogenic nutrient loading. While dynamics in ocean oxygen have strong feedbacks in the Earth system the precise relationships between ocean oxygenation state, geophysical forcing, global biogeochemical cycles, and climate are not well resolved. Notably, the controls on the development of ferruginous (iron-rich) versus euxinic (sulfide-rich) chemical states, and the corresponding implications for nutrient cycling are poorly known. Models that aim to mechanistically link ocean redox state to broader features of the Earth system are thus insufficiently constrained. In this talk, I will present an updated framework describing the fundamental controls on development of ferruginous versus euxinic conditions during ocean anoxia at different times in Earth's history. I'll also attempt to place new insight into nutrient cycling under low oxygen conditions into the broader context of these chemical states with corresponding implications for oceanic and global redox budgets.
\end{abstract}

